\title{
Evaluation of the differential capacitance for ferroelectric materials using either charge-based or energy-based expressions
}

\author{
C. M. Krowne \\ Electromagnetic Technology Branch \\ Electronics Science \& Technology Division \\ Naval Research Laboratory, Washington, D.C. 20375, USA \\ cliff.krowne@nrl.navy.mil
}

Received 7 May 2014; Revised 6 August 2014; Accepted 24 August 2014; Published 24 September 2014

\begin{abstract}
Differential capacitance is derived based upon energy, charge or current considerations, and determined when it may go negative or positive. These alternative views of differential capacitances are analyzed, and the relationships between them are shown. Because of recent interest in obtaining negative capacitance for reducing the subthreshold voltage swing in field effect type of devices, using ferroelectric materials characterized by permittivity, these concepts are now of paramount interest to the research community. For completeness, differential capacitance is related to the static capacitance, and conditions when the differential capacitance may go negative in relation to the static capacitance are shown.
\end{abstract}

Keywords: Differential capacitance; energy storage; ferroelectric dielectric material; negative capacitance; subthreshold voltage swing; reducing size of electronic devices; microscopic electric polarization; macroscopic permittivity.

\section{Introduction}

Our interest here is to understand how the differential capacitance, also called the small signal capacitance, or the ac capacitance, appears under different views. Three views are adopted, one being energy, the other two being charge and current, when the capactive material is characterizable by a permittivity. The motivation comes from the concern regarding the attributes of certain materials, particularly ferroelectric materials, for allowing negative capacitance in solidstate electronic devices. There has been past work examining negative capacitance, ${ }^{1-3}$ as well as recent interest ${ }^{4,5}$ related to the subthreshold voltage swing in FET type of devices utilizing insulating oxides to separate the metal gate from the conductive channel. Typical transistor three terminal devices dependent upon gate oxides include MESFETs and MOSFETs. For example, in MOSFETs the thickness and material type can significantly affect the interaction of the electrons with either bulk or surface phonons in the thin quantum sized $2 \mathrm{D}$ electron gas region ${ }^{6,7}$ where energy relaxation is closely related to energy storage issues. The more energy lost to the bulk and surface phonons just below the oxide-semiconductor interface, the worse the device performance. Furthermore, the size quantization of the electron gas due to the triangular shaped quantum well caused by the gate voltage applied across the oxide layer, will break up the electron band structure into subbands. Electron-phonon scattering results in electron transfer between subbands, with consequent power loss. ${ }^{8} 9$ MOSFET devices are of great interest presently in mono atomic (such as $\mathrm{Si}$ ) and compound semiconductor devices (various binary and ternary III-V materials for microwave or digital applications), ${ }^{10-21}$ so one sees that the oxide layer is a very critical feature of the transister.

Although the idea of a negative capacitance seems alien, it is worthwhile to recognize that negativity in physical device characteristics has been seen and utilized advantageously. One such area is negative differential resistivity (NDR), ${ }^{22,23}$ where two-terminal devices like solid-state diodes, can allow the extraction of ac energy from various structures like Esaki p-n diodes which obtain NDC through quantum tunneling between the valence and conduction bandgap, $\mathrm{n}^{+} \mathrm{nn}^{+}$Gunn or TED devices which rely on electron transfer to a higher conduction band with larger effective mass than the lower conduction band, or resonant tunneling devices (RTDs) which employ controlled transport through quantum barriers and quantum wells. Another area, extremely prominent recently, is the phenomenon of negative refraction (NR) or negative index materials (NIMs), whereby the electromagnetic wave can display a negative phase velocity, ${ }^{24}$ a reversed magnetic field circulation pattern and some negative Poynting (power) vector, ${ }^{25}$ a negative angle of refraction, ${ }^{26,27}$ and simultaneous negative permittivity and permeability which assures NR and NIM. ${ }^{28}$

In an earlier study,${ }^{29}$ the energy view was adopted with detailed discussions of Gibbs free energy $G$ versus electric polarization $P$, derivative of it $d G / d P$ with respect to $P$ versus $P$, and polarization $P$ versus electric field $E$. However, neglecting other viewpoints creates some confusion as to whether or not one viewpoint or another could allow a different boundary surface between negative and positive differential capacitance. This is particularly important to address

This is an Open Access article published by World Scientific Publishing Company. It is distributed under the terms of the Creative Commons Attribution 3.0 (CC-BY) License. Further distribution of this work is permitted, provided the original work is properly cited. 
at this point in time because in Ref. 29 the false claim in Ref. 4 that ferroelectric material could provide intrinsic negative differential capacitance was dispelled. Nevertheless, some of the same researchers have in Ref. 30 embedded a ferroelectric material in a dielectric insulator stack on an electronic device, and once again asserted experimentally that this shows ferroelectric negative capacitance. Of course, this is quite untrue, because Gibbs free energy and stability considerations cannot be violated as discussed in Ref. 29, and as shown herein other differential capacitance viewpoints do not admit a negative differential capacitance by extracting data from Ref. 29, and subjecting it to these alternative viewpoints.

We will not devote any attention here to many legitimate studies of negative capacitance arising from transient physical phenomena, such as spatial or momentum lag of carriers, trapping, and impact ionization, for example see Ref. 31, or discussed in other reviews or studies such as Refs. 32 and 33. The investigation here takes place in the larger context of finding ever smaller devices and material patterns to shrink active and control devices, partly determined and affected by charge storage and capacitance issues, occurring presently in elemental and binary, ternary and quaternary hetrostructure devices, carbon nanotube and graphene devices, and other nanowire and nanostructure devices. ${ }^{34-38}$ For completeness, differential capacitance is related to the static capacitance in Appendix A, and conditions when the differential capacitance may go negative in relation to the static capacitance are shown.

\section{Energy Differential Capacitance}

Therefore, let us begin by stating that the charge $Q$ on a capacitor with capacitance $C_{d}$ and a voltage across it of $V_{d}$ is given by

$$
Q=C_{d} V_{d}
$$

with the potential energy contained within it stated as ${ }^{39,40}$

$$
U=\frac{1}{2} \int_{\mathrm{Vol}} \varepsilon_{d} E_{d}^{2} d \tau=\frac{A_{d} s_{d}}{2} \varepsilon_{d}\left(\frac{V_{d}}{s_{d}}\right)^{2}=\frac{1}{2} C_{d} V_{d}^{2}
$$

Here $\varepsilon_{d}, s_{d}$, and $A_{d}$, are respectively the material permittivity, distance between conductive surfaces, and area of the capacitor. Equation (2) assumes $E_{d}$ field uniformity, a reasonable approximation which neglects edge fringing fields, especially valid when $\sqrt{A_{d}} / s_{d} \gg 1$.

Because the energetic considerations previously were what dominated the experimental measurements, as well as the theoretical modeling, power measurements (via magnitude squared values of the s-parameters) were made to determine an energy-based capacitance $C_{d}=C_{W}$. Thus (2) becomes the defining basis of the capacitance being first studied here. An ac or sinusoidal current basis of capacitance will be covered next in Sec. 3, and a charge basis of capacitance in Sec. 4.
If we demand that the energy $U$ be positive, ${ }^{41}$ as it must be for ordinary charge storage, $U>0$, then (2) implies

$$
C_{d}>0 \text {. }
$$

The displacement field $D_{d}$ is ${ }^{40,41}$

$$
\begin{aligned}
D_{d} & =\varepsilon_{0} E_{d}+P_{d}=\varepsilon_{0} E_{d}+\varepsilon_{0} \chi_{d} E_{d} \\
& =\varepsilon_{0}\left(1+\chi_{d}\right) E_{d}=\varepsilon_{d} E_{d} .
\end{aligned}
$$

In (4), $\varepsilon_{0}, \chi_{d}$ and $P_{d}$, are respectively the vacuum permittivity, electric susceptibility, and polarization field. Invoking $C_{d}=\left(A_{d} / s_{d}\right) \varepsilon_{d}$, yields utilizing (4),

$$
\varepsilon_{0}\left(1+\chi_{d}\right)>0 \text {. }
$$

This may also be written as

$$
\chi_{d}>-1
$$

which in terms of polarization and electric fields reads

$$
P_{d} / \varepsilon_{0} E_{d}>-1
$$

However, a different outcome ensues if we do not demand energy storage, the outcome of which was (3), but rather allow the supply of energy, which is negative energy storage, something a conventional battery would do. Of course, a capacitor is not a battery, so that perhaps one could envision an operating point at which no more stored energy exists in the structure $(U=0)$ and further energy removal correponds to $C_{d}$ going negative. In such a case $U<0$, and

$$
C_{d}<0
$$

Using (4) again, we must replace (6) by

$$
\chi_{d}<-1 \text {. }
$$

Let us now turn our attention to differential capacitance. Referring to (2), a differential stored energy element is (work $W$ is done to store energy $U$, thereby making $d W=d U$, besides $W=U$ )

$$
\begin{aligned}
d W & =\frac{1}{2} d\left(C_{d} V_{d}^{2}\right)=C_{d} V_{d} d V_{d}+\frac{1}{2} V_{d}^{2} d C_{d} \\
& =C_{d} V_{d} d V_{d}+\frac{A_{d}}{2 s_{d}} V_{d}^{2} \frac{d \varepsilon_{d}\left(V_{d}\right)}{d V_{d}} d V_{d} . \\
& =\left[1+\frac{1}{2} V_{d} \frac{1}{\varepsilon_{d}} \frac{d \varepsilon_{d}\left(V_{d}\right)}{d V_{d}}\right] C_{d} V_{d} d V_{d}
\end{aligned}
$$

It is a well-known phenomena in ferroelectric materials, for example, that material permittivity $\varepsilon_{d}$ is highly dependent on the local spatial electric field $E_{d}=V_{d} / s_{d}$ in the crystal. Therefore, the second term within the bracketed factor in the last equality of (10), dependent on the derivative $d \varepsilon_{d}\left(V_{d}\right) / d V_{d}$, may be nonzero and contribute. Now obtain from (10) the result for $d W$, with $C_{d}$ being invariant with voltage $V_{d}$, giving

$$
d W_{\text {const }, C_{d}}=C_{d} V_{d} d V_{d}
$$


Comparing (10) and (11), one can identify the bracketed term times $C_{d}$ in (10) as a capacitance, which is defined as the differential energy capacitance, given by

$$
\begin{aligned}
C_{\Delta W, d} & =\left[1+\frac{1}{n} V_{d} \frac{1}{\varepsilon_{d}} \frac{d \varepsilon_{d}\left(V_{d}\right)}{d V_{d}}\right] C_{d} \\
& =C_{d}+\frac{1}{n} \frac{d C_{d}\left(V_{d}\right)}{d V_{d}} V_{d},
\end{aligned}
$$

where we have generalized this expression by replacing the divisor 2 by $n$. The relationship between the differential energy capacitance and the ac capacitance will be determined below in Sec. 3. The ac capacitance may also be thought of as a sinusoidal-based current capacitance and its value of $n$ differs from that of the differential energy capacitance. Naturally, another type of capacitance arises from a charge basis with $C_{d}=C_{Q}$, generating a charge differential capacitance, which is treated in Sec. 4 , and gives a $C_{\Delta Q, d}$ in place of (12) also with $n \neq 2$.

Negative differential energy capacitance $C_{\Delta W, d}$ occurs when

$$
C_{\Delta W, d}<0
$$

or using (12),

$$
\left[1+\frac{1}{n} V_{d} \frac{1}{\varepsilon_{d}} \frac{d \varepsilon_{d}\left(V_{d}\right)}{d V_{d}}\right] C_{d}<0 .
$$

Due to (3), (14) becomes

$$
1+\frac{1}{n} V_{d} \frac{1}{\varepsilon_{d}} \frac{d \varepsilon_{d}\left(V_{d}\right)}{d V_{d}}<0
$$

or

$$
\frac{V_{d}}{\varepsilon_{d}} \frac{d \varepsilon_{d}\left(V_{d}\right)}{d V_{d}}<-n ; \quad n=2 .
$$

However, this may be restated in terms of the electric susceptibility, recalling (4),

$$
\frac{V_{d}}{1+\chi_{d}} \frac{d \chi_{d}\left(V_{d}\right)}{d V_{d}}<-n ; \quad n=2 .
$$

\section{AC Differential Capacitance}

The ac capacitance may also be thought of as a sinusoidalbased current capacitance, as mentioned above. Here will be shown the distinguishing characteristics between the differential energy capacitance $C_{\Delta W, d}$ and the ac capacitance $C_{\mathrm{ac}, d}$, which will be shortly derived. To do this, consider the current and voltage to be composed of a dc part and an ac part ${ }^{42}$

$$
\begin{aligned}
i(t) & =I_{\mathrm{dc}}+I_{\mathrm{ac}} \sin \left(\omega t+\varphi_{I}\right), \\
v(t) & =V_{\mathrm{dc}}+V_{\mathrm{ac}} \sin \left(\omega t+\varphi_{V}\right) .
\end{aligned}
$$

Then the ac capacitance $C_{\mathrm{ac}, d}$ relates the current through the capacitor and the rate of change of the voltage across it, namely $i_{C}(t)$ and $v_{C}(t)$ by

$$
i_{C}(t)=C_{\mathrm{ac}, d} \frac{d v_{C}(t)}{d t},
$$

where the ac parts of the current and voltage from (18) are

$$
\begin{gathered}
i_{C}(t)=I_{\mathrm{ac}} \sin \left(\omega t+\varphi_{I}\right), \\
v_{C}(t)=V_{\mathrm{ac}} \sin \left(\omega t+\varphi_{V}\right) .
\end{gathered}
$$

Place Eqs. (20a) and (20b) into (19),

$$
\begin{aligned}
I_{\mathrm{ac}} \sin \left(\omega t+\varphi_{I}\right) & =C_{\mathrm{ac}, d} \frac{d\left[V_{\mathrm{ac}} \sin \left(\omega t+\varphi_{V}\right)\right]}{d t} \\
& =\omega C_{\mathrm{ac}, d} V_{\mathrm{ac}} \cos \left(\omega t+\varphi_{V}\right) \\
& =\omega C_{\mathrm{ac}, d} V_{\mathrm{ac}} \sin \left(\omega t+\varphi_{V}+\pi / 2\right)
\end{aligned}
$$

which shows that the phase of the current through the ac capacitance is advanced by $\pi / 2$ or $90^{\circ}$ from the voltage across the ac capacitance.

$$
\phi_{I}=\phi_{V}+\pi / 2 .
$$

The ac capacitance itself, could be expanded about the total voltage seen by the capacitor, although, strictly speaking, this is not quite correct, because the ac capacitance relates the ac current amplitude $I_{\mathrm{ac}}$ to the voltage amplitude $V_{\mathrm{ac}}$ through the radian frequency $\omega$,

$$
I_{C}=\omega C_{\mathrm{ac}, d} V_{\mathrm{ac}} .
$$

Therefore, it is more correct to consider $C_{\mathrm{ac}, d}=C_{\mathrm{ac}, d}\left(V_{d}\right.$; $V_{\mathrm{ac}}$ ), and to maintain linearity in (23), $C_{\mathrm{ac}, d} \approx C_{\mathrm{ac}, d}\left(V_{d}\right)$, allowing a Taylor expansion about $V_{d}=V_{d 0},{ }^{43}$

$$
C_{d}\left(V_{d}\right)=\left.\sum_{n=0}^{\infty} \frac{1}{n !} \frac{d^{n} C_{d}\left(V_{d}\right)}{d^{n} V_{d}}\right|_{V_{d}=V_{d 0}}\left(V_{d}-V_{d 0}\right)^{n} .
$$

Keeping the first two terms of the series,

$$
C_{d}\left(V_{d}\right)=C_{d 0}+\left.\frac{d C_{d}\left(V_{d}\right)}{d V_{d}}\right|_{V_{d}=V_{d 0}}\left(V_{d}-V_{d 0}\right) .
$$

Here $C_{d 0}=C_{d}\left(V_{d 0}\right) . V_{d 0}$ may be taken to correspond as in (1) to stored charge, in which case, when $V_{d 0}=0$, and (25) becomes

$$
C_{d}\left(V_{d} ; V_{d 0}=0\right)=C_{0}+\left.\frac{d C_{d}\left(V_{d}\right)}{d V_{d}}\right|_{V_{d}=0} V_{d} .
$$

Here $C_{0}=C_{d}\left(V_{d 0}=0\right)$. However, to do a comparison directly with the differential energy capacitance given by (12), $V_{d 0}$ must be finite, necessitating reaarangement of (25),

$$
\begin{aligned}
C_{d}\left(V_{d}\right)= & C_{d 0}-\left.\frac{d C_{d}\left(V_{d}\right)}{d V_{d}}\right|_{V_{d}=V_{d 0}} V_{d 0} \\
& +\left.\frac{d C_{d}\left(V_{d}\right)}{d V_{d}}\right|_{V_{d}=V_{d 0}} V_{d} \\
= & C_{d}+\left.\frac{d C_{d}\left(V_{d}\right)}{d V_{d}}\right|_{V_{d}=V_{d 0}} V_{d}
\end{aligned}
$$


with $C_{d}$ defined as

$$
C_{d}=C_{d 0}-\left.\frac{d C_{d}\left(V_{d}\right)}{d V_{d}}\right|_{V_{d}=V_{d 0}} V_{d 0} .
$$

The capacitance appearing in (27) may be identified with the ac capacitance that appears in (23), or

$$
C_{\mathrm{ac}, d}=C_{d 0}+\left.\frac{d C_{d}\left(V_{d}\right)}{d V_{d}}\right|_{V_{d}=V_{d 0}} V_{d} .
$$

Dropping the subscript using "0", it being understood, (29) reduces to

$$
C_{\mathrm{ac}, d}=C_{d}+\frac{d C_{d}\left(V_{d}\right)}{d V_{d}} V_{d}
$$

restricting $n=1$ in the generalized equation (12), which was derived originally in the differential energy capacitance analysis. However, the second equality in (12) for differential energy capacitance is

$$
C_{\Delta W, d}=C_{d}+\frac{1}{2} \frac{d C_{d}\left(V_{d}\right)}{d V_{d}} V_{d} .
$$

Therefore, the difference between the ac and differential energy capacitances is

$$
C_{\mathrm{ac}, d}-C_{\Delta W, d}=\frac{1}{2} \frac{d C_{d}\left(V_{d}\right)}{d V_{d}} V_{d} .
$$

When inserted into (31),

$$
C_{\Delta W, d}=C_{d}+C_{\mathrm{ac}, d}-C_{\Delta W, d}
$$

making

$$
C_{\Delta W, d}=\frac{1}{2} C_{d}+\frac{1}{2} C_{\mathrm{ac}, d} .
$$

For the ac differential capacitance to be negative

$$
C_{\mathrm{ac}, d}<0
$$

or

$$
C_{d}+\frac{d C_{d}\left(V_{d}\right)}{d V_{d}} V_{d}<0
$$

or in terms of permittivity,

$$
\frac{d \varepsilon_{d}\left(V_{d}\right)}{d V_{d}}<-\frac{\varepsilon_{d}}{V_{d}}
$$

showing that $n=1$ here.

Figure 1 demonstrates Eqs. (16) and (37) for, respectively, the energy and current viewpoints, showing the negative differential capacitance condition for ferroelectric material studied in Ref. 29. The material is $\mathrm{SrTiO}_{3}$, an incipient ferroelectric, which acts as a paraelectric material down to $0 \mathrm{~K}$. Application of strain to the films was utilized to create ferroelectric behavior. Table 1 shows a variety of commonly used ferroelectric materials (other materials listed or discussed in Refs. 44-47), four inorganic $\left(\mathrm{ABO}_{3}\right.$ generic

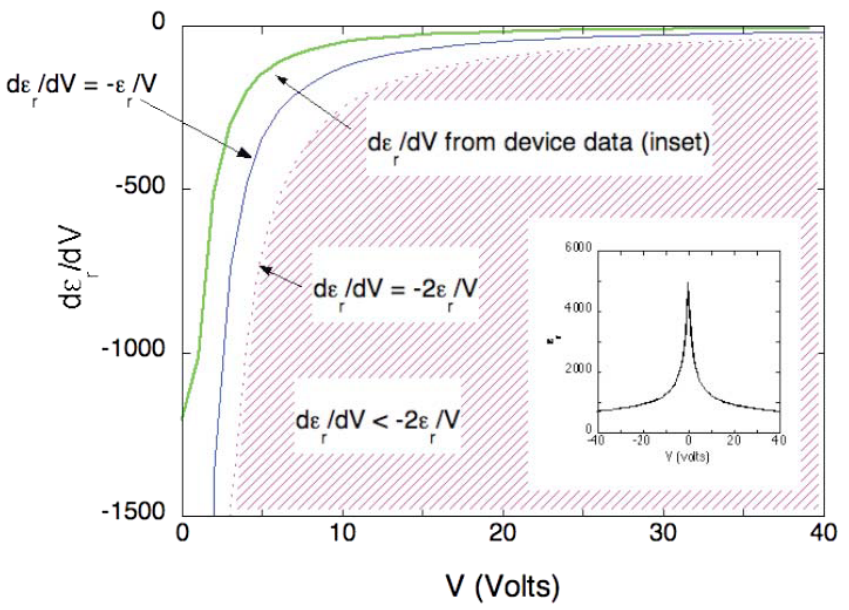

Fig. 1. (Color online) Plot of the derivative of the relative dielectric constant versus voltage, $d \varepsilon_{d} / d V_{d}$ versus $V_{d}$, for data displayed in inset curve $\varepsilon_{d}$ versus $V_{d}$, along with the corresponding regions satisfying inequalities (16) [the cross-hatched pink region to the right of the pink dotted boundary line $\left.d \varepsilon_{r} / d V=-2 \varepsilon_{r} / V\right]$, and (37)/(44) [the cross-hatched pink region and the region to the right of the solid blue boundary line $d \varepsilon_{r} / d V=-\varepsilon_{r} / V$ and to the left of the pink dotted boundary line $\left.d \varepsilon_{r} / d V=-2 \varepsilon_{r} / V\right]$. The inset curve represents measured data first reported in Ref. 49.

perovskite formula) and one organic, including the one utilized in Fig. 1. Table 1 entries include name, chemical formula and unit cell dimensions, Curie temperature $T_{C}(\mathrm{~K})$, density $\left(\mathrm{g} / \mathrm{cm}^{3}\right)$, crystal structure, crystallographical group properties (space or point group; Hermann-Mauguin or Schoenflies notation; here $\underline{3}=\overline{3}),{ }^{48}$ and remanent $P_{r}$ or spontaneous $P_{s}$ (approximately equal to saturated polarization $P_{\text {sat }}, P_{s} \leq P_{\text {sat }}$ ) polarization. It is seen that the line separating the negative from positive negative differential capacitance changes when going from the current viewpoint (blue line, $n=1$ ) to an energy viewpoint (dotted pink line, $n=2$ ). The line representing the energy viewpoint is to the right of the current viewpoint, and both are to the right of the actual device data, shown by the solid green line. Thus, in no case does the ferroelectric differential capacitance in actual devices ever go negative.

\section{Differential Charge Capacitance}

Charge-based differential capacitance may be obtained in the following manner. Retrieve relationship (1),

$$
Q=C_{d} V_{d} .
$$

This type of capacitance arrises from a charge basis with $C_{d}=C_{Q}$, generating a charge differential capacitance. Now, the differential charge is

$$
d Q=V_{d} \frac{d C_{d}\left(V_{d}\right)}{d V_{d}} d V_{d}+C_{d} d V_{d} .
$$




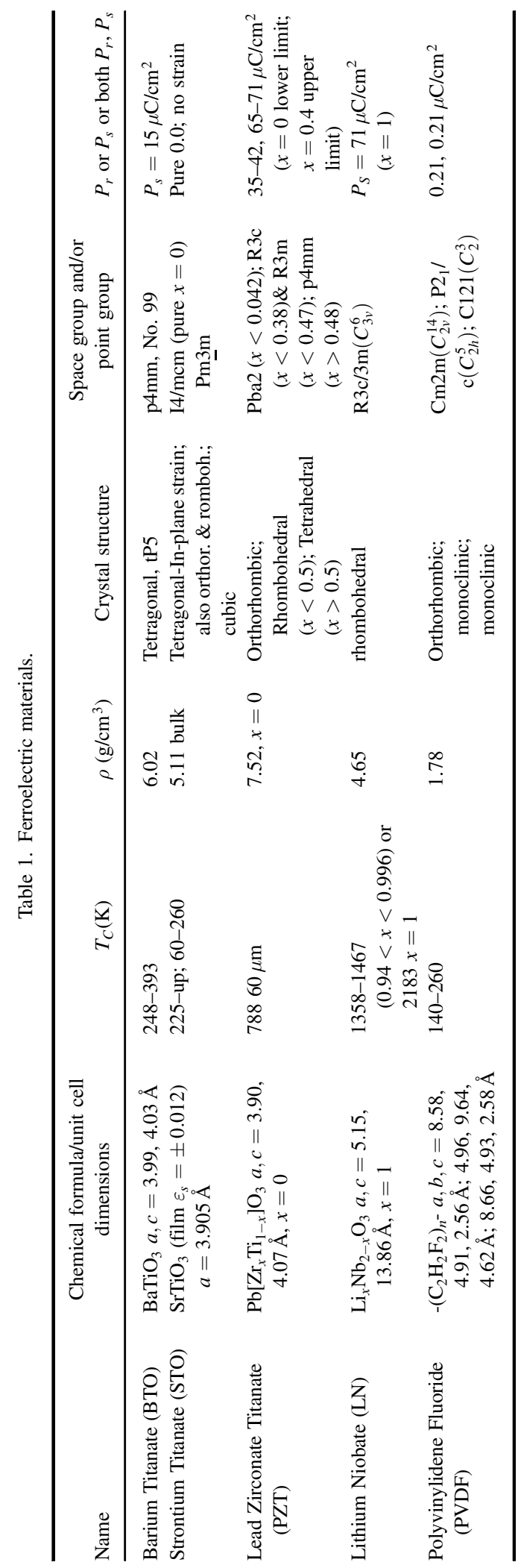


Differential work done by separating an extra element of charge $d Q$ through a potential difference element $d V_{d}$ is

$$
d W=V_{d} d Q .
$$

Inserting $d Q$ from (39) into $d W$ of (41) yields,

$$
\begin{aligned}
d W & =V_{d}\left[V_{d} \frac{d C_{d}\left(V_{d}\right)}{d V_{d}} d V_{d}+C_{d} d V_{d}\right] \\
& =\left[V_{d} \frac{d C_{d}\left(V_{d}\right)}{d V_{d}}+C_{d}\right] V_{d} d V_{d} \\
& =\left[1+V_{d} \frac{1}{\varepsilon_{d}} \frac{d \varepsilon_{d}\left(V_{d}\right)}{d V_{d}}\right] C_{d} V_{d} d V_{d}
\end{aligned}
$$

and we may identify

$$
\begin{aligned}
C_{\Delta Q, d} & =\left[1+V_{d} \frac{1}{\varepsilon_{d}} \frac{d \varepsilon_{d}\left(V_{d}\right)}{d V_{d}}\right] C_{d} \\
& =C_{d}+\frac{d C_{d}\left(V_{d}\right)}{d V_{d}} V_{d}
\end{aligned}
$$

as the charge-based differential capacitance. Clearly, upon examining (29) and (42), the ac differential capacitance and the charge-based differential capacitance are identical,

$$
C_{\mathrm{ac}, d}=C_{\Delta Q, d} .
$$

Equation (43) combined with (36) yields

$$
\frac{d \varepsilon_{d}\left(V_{d}\right)}{d V_{d}}<-\frac{\varepsilon_{d}}{V_{d}}
$$

again.

Now Fig. 1 demonstrates Eqs. (16), (37) and (44) for, respectively, the energy, current and charge viewpoints, showing the negative differential capacitance condition for ferroelectric material. It is seen that the line separating the negative from positive differential capacitance changes when going from the current or charge viewpoints (blue line, $n=1$ ) to an energy viewpoint (dotted pink line, $n=2$ ). The line representing the energy viewpoint is to the right of the current and charge viewpoints, and both are to the right of the actual device data, shown by the solid green line. Thus, again in no case does the ferroelectric differential capacitance in actual devices ever go negative.

\section{Conclusions}

In summary, what we have learned is that there is a basic constraint equation that determines when a material characterized by a permittivity, has its differential capacitance go negative, and that for a ferroelectric material, ${ }^{49-65}$ no matter what the viewpoint, energy or current (or charge), this value can never be negative and that this may occur for either sign of the dc capacitance (see Appendix A). Furthermore, there is another similar constraint equation for obtaining positive differential energy capacitance, and that this, as before, may occur for either sign of the dc capacitance. These results have direct bearing on all investigations pursuing negative capacity capability using basic material properties of substances. All substances in Table 1, commonly used ferroelectric materials, have the ability in principle to take excursions into the regions of the polarization $P$ versus electric field $E$ hysteresis curve where $d P / d E<0$ (see Fig. 3(b) of Ref. 29), and generate negative differential capacitance, but due to energetic considerations, this is extremely unlikely, as demonstrated by the Gibbs free energy $U$ and its derivative versus $P$ (see Figs. 2 and 3(a) of Ref. 29 for $\mathrm{BaTiO}_{3}$ ).

Because this paper has only examined capacitances, its scope not including resistances and their associated losses, it should be noted that in general, hysteresis loops occur in parts of the four $P-E$ (or $V-I$ ) quadrants. The quadrants where $P E<0$ (or $V I<0$ ) will correspond to energy supplied, and that where $P E>0$ (or $V I>0$ ) will be energy taken. For a system like ferroelectric, most of that energy will be of the reactive type, although imperfect ferroelectric materials have some dissipative loss which will add to the energy balance sheet in terms of raising the subthreshold limit. Since for an ideal ferroelectric material the hysteresis curve is symmetric about the $P-E$ origin, there is no net gain or loss of reactive energy after completion of one cycle of the hysteresis loop. In a circuit characterized by an $I-V$ hysteresis loop, if that loop is off center, then a net energy loss might occur, with attendant dissipation. Completing a full cycle around a hysteresis loop for reactive energy cost requires consideration of the signs of $P$ and $E$. For dissipative energy cost, one will generally need more information than the $P-E$ hysteresis loop, and one must not succumb to the notion that the loop area is directly proportional to the dissipative loss. This can be easily shown to be false for the illustrative case of a simple ideal resistor, whose $V-I$ curve is a straight line from the origin. Consider it to be a hysteresis loop of very small area, so that the movement from $I=0$ to $I=I_{\max }$ has the loop bowed down, below the $V=I R$ line, and the return loop from $I=$ $I_{\max }$ to $I=0$ is bowed up, above the $V=I R$ line. Clearly the dissipated energy is related to the integral in time of the area under the line and not the loop area, as the bowing is allowed to become infinitely thin.

\section{Acknowledgment}

The author thanks Dr. Steven W. Kirchoefer of NRL for producing the plot in Fig. 1, by changing the integer dividing factor in the denominator of the differential capacitance expression.

\section{Appendix A}

Examine condition (13), using the last expression in (12),

$$
\frac{1}{n} \frac{d C_{d}}{C_{d}}<-\frac{d V_{d}}{V_{d}}, \quad C_{d}>0
$$


and form the integral inequality equation

$$
\frac{1}{n} \int \frac{d C_{d}}{C_{d}}<-\int \frac{d V_{d}}{V_{d}}, \quad C_{d}>0 .
$$

Performing the integrations in (A.2) gives

$$
\frac{1}{n} \ln C_{d}+A_{C}<-\ln V_{d}+A_{V}
$$

or

$$
C_{d}^{1 / n}<\frac{B_{V}}{B_{C}} \frac{1}{V_{d}}, \quad C_{d}>0, \quad C_{\Delta W, d}<0,
$$

where $B_{C}=e^{A_{C}}$ and $B_{V}=e^{A_{V}}, A_{C}$ and $A_{V}$ are the integration constants $\left[A_{C}=(-1 / n) \ln C_{d}\left(e^{A_{v}}\right)\right.$ or $\left.B_{C}=C_{d}^{-1 / n}\left(B_{V}\right)\right]$. (Integrate between upper and lower capacitance values $C_{d u}$ and $C_{d l}$ and the corresponding voltages $V_{d u}$ and $V_{d l}$, and set $C_{d u} \rightarrow C_{d}, \quad V_{d u} \rightarrow V_{d}$ and identify $A_{C}=-(1 / n) \ln \left(C_{d l}\right)$, $A_{V}=\ln \left(V_{d l}\right)$.) The positive voltage branch $V_{d}>0$ is used.

What transpires if $C_{d}<0$ ? Go back to the inequality constraint (13), again referring to (12),

$$
\frac{1}{n} \frac{d C_{d}}{C_{d}}>-\frac{d V_{d}}{V_{d}}, \quad C_{d}<0 .
$$

In order to avoid handling the natural log of a negative capacitance, we choose for convenience to work with the magnitude of $C_{d}$, or $C_{m d}=\left|C_{d}\right|$.

$$
\frac{1}{n} \frac{d C_{d m}}{C_{d m}}>-\frac{d V_{d}}{V_{d}}, \quad C_{d}<0
$$

Integrating (A.6) generates

$$
\frac{1}{n} \ln C_{d m}+A_{C m}>-\ln V_{d}+A_{V m} .
$$

By (A.7), the solution becomes

$$
C_{d m}^{1 / n}>\frac{B_{V m}}{B_{C m}} \frac{1}{V_{d}}, \quad C_{d}<0, \quad C_{\Delta W, d}<0,
$$

where $B_{C m}=e^{A_{C m}}$ and $B_{V m}=e^{A_{V m}}, A_{C m}$ and $A_{V m}$ are the integration constants $\left[A_{C m}=(-1 / n) \ln C_{d m}\left(e^{A_{V m}}\right)\right.$ or $B_{C m}=$ $\left.C_{d m}^{-1 / n}\left(B_{V m}\right)\right]$.

From the above arguments, we see that a negative differential energy capacitance (or for that matter, a current or charge viewpoint, changing $n$ ) does not necessarily specify the dc capacitance. In fact, all that is required to have $C_{\Delta W, d}$ $<0$ is to have an overpowering $d C_{d} / d V_{d}$ derivative. Consider the following example. Let

$$
C_{d}=A\left(1-a V_{d}^{b}\right)
$$

in the range where

$$
a V_{d}^{b}<1, \quad A>0, \quad a>0 .
$$

In this range, $C_{d}>0$. Then $d C_{d} / d V_{d}=-a A b V_{d}^{b-1}$, making $C_{\Delta W, d}$ by (12)

$$
C_{\mathrm{ac}, d}=A-a A\left(1+\frac{b}{n}\right) V_{d}^{b} .
$$

To satisfy (13)

$$
A-a A\left(1+\frac{b}{n}\right) V_{d}^{b}<0
$$

or

$$
1-a\left(1+\frac{b}{n}\right) V_{d}^{b}<0
$$

which will always hold for

$$
b>n\left[\frac{1}{a V_{d}^{b}}-1\right] \text {. }
$$

For a general positive $b$ value, setting

$$
a=V_{d}^{-b-\delta}
$$

makes (A.10) require

$$
\left(V_{d}\right)^{-\delta}<1
$$

which is true for $V_{d}>1$ and $\delta>0$. Placing $a$ into (A.14), $\delta$ must obey

$$
b>n\left[\frac{1}{V_{d}^{-\delta}}-1\right]=n\left(V_{d}^{\delta}-1\right) .
$$

Rearranging this inequality yields

$$
\delta<\frac{\ln (b / n+1)}{\ln V_{d}} .
$$

The above arguments show that for a negative differential energy capacitance $C_{\Delta W, d}$, i.e., $C_{\Delta W, d}<0$, one could have either a positive or negative dc capacitance $C_{d}$. But suppose one had a positive $C_{\Delta W, d}$, what are the implications for $C_{d}$ ? Therefore,

$$
C_{\Delta W, d}>0 \text {. }
$$

Following the logic for the $C_{\Delta W, d}<0$ case, from (A.7) with appropriate modifications, we have

$$
-\frac{1}{n} \ln C_{d m}+A_{C m}>\ln V_{d}+A_{V m}
$$

with $A_{C m}=(1 / n) \ln C_{d m}\left(e^{-A_{V m}}\right)$ or $B_{C m}=C_{d m}^{1 / n}\left(B_{V m}^{-1}\right)$. From (A.20),

$$
C_{d m}^{-1 / n}>\frac{B_{V m}}{B_{C m}} V_{d}, \quad C_{d}<0, \quad C_{\Delta W, d}>0
$$

or

$$
\left|C_{d}\right|<R_{C V m} V_{d}^{-n}, \quad C_{d}<0, \quad C_{\mathrm{ac}, d}>0,
$$


where $R_{C V m}=\left(B_{C m} / B_{V m}\right)^{n}$. Make the assignment

$$
C_{d}=-R_{C V m} V_{d}^{-n-\delta}
$$

which causes

$$
\left(V_{d}\right)^{-\delta}<1
$$

by (A.21). Condition (A.24) is satisfied for $V_{d}>1$, so that is the range considered for this example. By (12)

$$
C_{\Delta W, d}=\frac{\delta}{n} R_{C V m} V_{d}^{-n-\delta}
$$

and to obey (A.19),

$$
\delta>0 .
$$

Thus, for positive $\delta$, (A.25) gives a positive $C_{\Delta W, d}$, satisfying (A.19). We conclude that it is quite feasible to have positive a differential energy capacitance (or differential charge capacitance - simply change $n$ from 2 to 1) while having a negative dc capacitance.

In summary, what we have learned is that there is a basic constraint equation that determines when a material characterized by a permittivity, has its differential energy (or sinusoidal current or charge) capacitance go negative, and that this may occur for either sign of the dc capacitance. Furthermore, there is another similar constraint equation for obtaining positive differential energy (or sinusoidal current or charge) capacitance, and that this, as before, may occur for either sign of the dc capacitance. These results have direct bearing on all investigations pursuing negative capacity capability using basic material properties of substances.

One final point should be made concerning the case when the dc capacitance is negative, This generally corresponds to, by (2), negative stored energy, and could involve nonequilibrium conditions. If in obtaining $C_{d}<0$ energy pumping is required, then this would have to be accounted for not just in subthreshold voltage change, but also in the switching energy (former implies latter). It is shown in another study of ferroelectric materials, ${ }^{29}$ however, that its dc capacitance is not negative, and that the differential energy capacitance never veers off into the negative region.

\section{References}

${ }^{1} \mathrm{R}$. Landauer, Can capacitance be negative? Collective Phenomena 2, 167 (1976).

${ }^{2}$ A. K. Jonscher, The physical origin of negative capacitance, J. Chem. Soc. Faraday Trans. 2 82, 75 (1986).

${ }^{3}$ S. A. Bhosale, G. S. Nadkarni and S. Radhakrishnan, Negative capacitance in thin film $\mathrm{Al}-\mathrm{V}_{2} \mathrm{O}_{5}-\mathrm{Al}$ devices, Phys. Stat. Sol. (a) 101, 639 (1987).

${ }^{4}$ S. Salahuddin and S. Datta, Use of negative capacitance to provide voltage amplification for low power nanoscale devices, Nano Lett. 8, 405 (2008).
${ }^{5}$ V. V. Zhirnov and R. K. Cavin, Negative capacitance to the rescue? Nat. Nanotech. 3, 77 (2008).

${ }^{6}$ C. M. Krowne and J. W. Holm-Kennedy, Energy relaxation of electrons in the (100) n-channel of a Si-MOSFET. I. Bulk phonon treatment, Surf. Sci. 46, 197 (1974).

${ }^{7}$ C. M. Krowne and J. W. Holm-Kennedy, Energy relaxation of electrons in the (100) n-channel of a Si-MOSFET. II. Surface phonon treatment, Surf. Sci. 46, 232 (1974).

${ }^{8}$ C. M. Krowne, Electron power loss in the (100) channel of a Si metal oxide-semiconductor field effect transistor. I. Intrasubband phonon scattering, J. Appl. Phys. 54, 2441 (1983).

${ }^{9}$ C. M. Krowne, Electron power loss in the (100) channel of a Si metal oxide-semiconductor field effect transistor. II. Intersubband phonon scattering, J. Appl. Phys. 54, 2455 (1983).

${ }^{10}$ H. Zhao, Y.-T. Chen, J. H. Yum, Y. Wang, F. Zhou, F. Xue and J. C. Lee, Effects of barrier layers on device performance of high mobility $\mathrm{In}_{0.7} \mathrm{Ga}_{0.3}$ As metal-oxide-semiconductor field effecttransistors, Appl. Phys. Lett. 96, 102101 (2010).

${ }^{11} \mathrm{~K}$. Natori, Ballistic metal-oxide-semiconductor field effect transistor, J. Appl. Phys. 76, 4879 (1994).

${ }^{12}$ Y. Q. Wu, P. D. Ye, G. D. Wilk and B. Yang, GaN metal-oxide semiconductor field-effect transistor with atomic layer deposited $\mathrm{Al}_{2} \mathrm{O}_{3}$ as gate dielectric, Mater. Sci. Eng. B 135, 282 (2006).

${ }^{13}$ Y. Dong, M. Chen, J. Chen, X. Wang, Xi Wang, P. He, Xi Lin, L. Tian and Z. Li, Patterned buried oxide layers under a single MOSFET to improve the device performance, Semicond. Sci. Tech. 19, L25 (2004).

${ }^{14}$ W. Liu, X. Jin, Y. King and C. Hu, An efficient and accurate compact model for thin-oxide-MOSFET intrinsic capacitance considering the finite charge layer thickness, IEEE Trans. Electron Devices 46, 1070 (1999).

${ }^{15}$ D. Rank, A. K. Rana, R. K. Yadav and D. Giri, Performance analysis of FD-SOI MOSFET with different gate spacer dielectric, Int. J. Comput. Appl. 18, 22 (2011).

${ }^{16}$ K. Roy, S. Mukhopadhyay and H. Mahmoodi-Meimand, Leakage current mechanisms and leakage reduction techniques in deepsubmicrometer CMOS circuits, Proc. IEEE 91, 305 (2003).

${ }^{17} \mathrm{P}$. Jena, R. Ku Mishra and K. H. Krishna, Comparative study of SOI-MOSFET modelling structure and their characterisation through simulation TCAD tool, Int. J. Eng. Res. Tech. 1 (2012) ISSN 2278-0181.

${ }^{18}$ V. A. Sverdlov, T. J. Walls and K. K. Likharev, Nanoscale silicon MOSFETs: A theoretical study, IEEE Trans. Electron Devices 50, 1926 (2003).

${ }^{19}$ S.-H. Chen, W.-S. Liao, H.-C. Yang, S.-J. Wang, Y.-G. Liaw, H. Wang, H. Gu and M.-Ch. Wang, High-performance III-V MOSFET with nano-stacked high-k gate dielectric and 3D finshaped structure, Nanoscale Res. Lett. 7, 431 (2012).

${ }^{20}$ M. Zhu, P. Chen, R. K.-Y. Fu, Z. An, C. Lin and P. K. Chu, Numerical study of self-heating effects of MOSFETs fabricated on SOAN substrate, IEEE Trans. Electron Devices 51, 901 (2004).

${ }^{21}$ V. Kumari, M. Saxena, R. S. Gupta and M. Gupta, Investigation of empty space in nanoscale double gate (ESDG) MOSFET for high speed digital circuit applications, J. Semicond. Tech. Sci. 13, 127 (2013).

${ }^{22}$ C. M. Krowne and P. Blakey, On the existence of submillimeterwave negative conductance in n-gallium arsenide diodes, J. Appl. Phys. 61, 2257 (1987). 
${ }^{23}$ C. M. Krowne, Negative resistance and distributed gain in submicron semiconductor diodes using analytical solutions of the Boltzmann transport equation, Int. J. Electron. 62, 483 (1987).

${ }^{24}$ A. Lakhtakia and C. M. Krowne, Restricted equivalence of paired epsilon-negative and mu-negative layers to a negative phasevelocity material (alias left-handed material), Optik 114, 305 (2003).

${ }^{25}$ C. M. Krowne, Physics of propagation in left-handed guided wave structures at microwave and millimeter wave frequencies, Phys. Rev. Lett. 92, 053901 (2004).

${ }^{26}$ C. M. Krowne, Negative refractive bicrystal with broken symmetry produces asymmetric electromagnetic fields in guided-wave heterostructures, Phys. Rev. Lett. 93, 053902 (2004).

${ }^{27}$ C. M. Krowne, Negative refractive heterostructure bicrystal for beam steering or control component action, Appl. Phys. Lett. 91, 022902 (2007).

${ }^{28}$ C. M. Krowne and J. Q. Shen, Dressed-state mixed-parity transitions for realizing negative refractive index, Phys. Rev. A 93, 023818 (2009).

${ }^{29}$ C. M. Krowne, S. W. Kirchoefer, W. Chang, J. M. Pond and L. M. B. Alldredge, Examination of the possibility of negative capacitance using ferroelectric materials in solid state devices, Nano Lett. 11, 988 (2011).

${ }^{30}$ A. I. Khan, D. Bhowmik, P. Yu, S. J. Kim, X. Pan, R. Ramesh and S. Salahuddin, Experimental evidence of ferroelectric negative capacitance in nanoscale heterostructures, Appl. Phys. Lett. 99, 113501 (2011).

${ }^{31}$ T. Kopp and J. Mannhart, Calculation of the capacitances of conductors: Perspectives for the optimization of electronic devices, J. Appl. Phys. 106, 064504 (2009).

${ }^{32}$ L. Li, C. Richter, S. Paetel, T. Kopp, J. Mannhart and R. C. Ashoori, Very large capacitance enhancement in a two-dimensional electron system, Science 332, 825 (2011).

${ }^{33}$ M. Ershov, H. C. Liu, L. Li, M. Buchanan, Z. R. Wasilewski and A. K. Jonscher, Negative capacitance effect in semiconductor devices, IEEE Trans. Electron Devices 45, 2196 (1998).

${ }^{34}$ C. M. Krowne, Intrinsic quantum conductances and capacitances of nanowire and nanocables, Phys. Lett. A 374, 614 (2010).

${ }^{35}$ C. M. Krowne, Nanowire and nanocable intrinsic quantum capacitances and junction capacitances: Results for metal and semiconducting oxides, J. Nanomater., Article ID 160639 (2011), doi: $10.1155 / 2010 / 160639$.

${ }^{36}$ B. D. Kong, Y. G. Semenov, C. M. Krowne and K. W. Kim, Unusual magnetoresistance in a topological insulator with a single ferromagnetic barrier, Appl. Phys. Lett. 98, 24311 (2011).

${ }^{37}$ J. I. Martinez, F. Calle-Vallejo, C. M. Krowne and J. A. Alonso, First-principles structural and electronic characterization of ordered $\mathrm{SiO}_{2}$ nanowires, J. Phys. Chem. C 116, 18973 (2012).

${ }^{38}$ J. I. Martinez, F. Calle-Vallejo, C. M. Krowne and J. A. Alonso, Tailoring structural and electronic properties of $\mathrm{RuO}_{2}$ nanotubes: Many-body approach and electronic transport, Phys. Chem. Chem. Phys. 15(35), 14715 (2013).

${ }^{39}$ E. M. Purcell, Electricity and Magnetism, Berkeley Physics Course, Vol. 2 (McGraw-Hill, New York, 1965).

${ }^{40}$ E. C. Jordan and K. G. Balmain, Electromagnetic Waves and Radiating Systems (Prentice-Hall, New Jersey, 1968).

${ }^{41}$ J. D. Jackson, Classical Electromagnetism (Wiley, New York, 1962).
${ }^{42}$ J. B. Cruz Jr. and M. E. Van Valkenburg, Introductory Signals and Circuits (Blaisdell Publishing, Waltham, MA, 1967).

${ }^{43}$ M. H. Protter and C. B. Morrey Jr., Modern Mathematical Analysis (Addison-Wesley Publishing, 1964).

${ }^{44}$ M. E. Lines and A. M. Glass, Principles and Applications of Ferroelectrics and Related Materials (Oxford Univ. Press, Oxford, 1977).

${ }^{45}$ D. K. Das-Gupta (ed.), Ferroelectric Polymers and CeramicPolymer Composites (TransTech Publ., Switzerland, 1994).

${ }^{46}$ L. Pardo and J. Ricote, Multifunctional Polycrystalline Ferroelectric Materials (Springer, The Netherlands, 2011).

${ }^{47}$ E. Defay (ed.), Ferroelectric Dielectrics Integrated on Silicon (Wiley, London, UK, 2011).

${ }^{48}$ M. N. Julian, Foundations of Crystallography with Computer Applications (CRC Press, 2008).

${ }^{49}$ J. H. Haeni, P. Irvin, W. Chang, R. Uecker, P. Relche, Y. L. Li, S. Choudhury, W. Tian, M. E. Hawley, B. Craigo, A. K. Tagantsev, X. Q. Pan, S. K. Streiffer, L. Q. Chen, S. W. Kirchoefer, J. Levy and D. G. Schlom, Room-temperature ferroelectricity in strained $\mathrm{SrTiO}_{3}$, Nature 430, 758 (2004).

${ }^{50}$ D. A. Barrow, T. E. Petroff, R. P. Tandon and M. Sayer, Characterization of thick lead zirconate titanate films fabricated using a new sol gel based process, J. Appl. Phys. 81, 876 (1997).

${ }^{51}$ N. Iyi, K. Kitamura, F. Izumi, J. K. Yamamoto, T. Hayashi, H. Aasno and S. Kimura, Comparative study of defect structure in lithium niobate with different compositions, J. Solid State Chem. 101, 340 (1992).

${ }^{52}$ A. J. Lovinger, D. D. Davis, R. E. Cais and J. M. Kotemani, On the Curie temperature of poly(vinylidene fluoride), Macromolecules 19, 1491 (1986).

${ }^{53}$ Y. L. Gal'Perin, Y. V. Strogalin and M. P. Mlenik, Crystal structure of polyvinylidene fluoride, Polymer Sci. U.S.S.R. 7, 1031 (1965).

${ }^{54}$ R. Hasegawa, Y. Takahashi, Y. Chatani and H. Tadokoro, Crystal structures of three crystalline forms of poly(vinylidene fluoride), Polymer J. 3, 600 (1972).

${ }^{55}$ J. Carvell and R. Cheng, Study of electrical polarization hysteresis in ferroelectric polyvinylidene fluoride films, Mater. Lett. 64, 1992 (2010).

${ }^{56}$ Z. J. Wang, Y. Aoki, L. J. Yan, H. Kokawa and R. Maeda, Crystal structure and microstructure of lead zirconate titanate (PZT) thin films with various $\mathrm{Zr} / \mathrm{Ti}$ ratios grown by hybrid processing, J. Crystal Growth 267, 92 (2004).

${ }^{57}$ Z. Surowiak, D. Czekaj, E. G. Fesenko, O. N. Razumovskaya, L. A. Reznichenko and I. N. Zakharchenko, Influence of the chemical composition on the physical properties of PZT-type piezoceramic transducers, Mol. Quant. Acoust. 24, 183 (2003).

${ }^{58}$ H. Nakaki, H. Uchida, S. Koda, S. Okamoto, H. Funakubo, K. Nishida, T. Katoda and K. Saito, Enhancement of spontaneous polarization in lead zirconate titanate thin films by Dy3+ substitution, Appl. Phys. Lett. 87, 182906 (2005).

${ }^{59}$ S. C. Abrahams, J. M. Reddy and J. L. Bernstein, Ferroelectric lithium niobate. 3. Single crystal X-ray difraction study at $24 \mathrm{C}, J$. Phys. Chem. Solids 27, 997 (1966).

${ }^{60}$ K. Nassau, H. J. Levinstein and G. M. Loiacono, Ferroelectric lithium niobate. 2. Preparation of single domain crystals, J. Phys. Chem. Solids 27, 989 (1966).

${ }^{61}$ S. H. Wemple, M. DiDomenico Jr. and I. Camlibel, Relationship between linear and quadratic electro-optic coefficients in $\mathrm{LiNbO} 3$, 
LiTaO3, and other oxygen-octahedra ferroelectrics based on direct measurement of spontaneous polarization, Appl. Phys. Lett. 12, 209 (1968).

${ }^{62}$ B. Wodecka-Dus and D. Czekaj, Fabrication and dielectric properties of donor doped $\mathrm{BaTiO}_{3}$ ceramics, Arch. Metall. Mater. 54, 923 (2009).

${ }^{63}$ A. G. H. Smith, Structural and defect properties of strontium titanate, Ph.D. Dissertation, Department of Chemistry, University College London (2011).
${ }^{64}$ V. A. Chaudhari and G. K. Bichile, Synthesis, structural, and electrical properties of pure $\mathrm{PbTiO}_{3}$ ferroelectric ceramics, Smart Mater. Res., 2013, Art. ID 147524 (2013) 9 pp.

${ }^{65}$ C. A. Randall, N. Kim, J.-P. Kucera, W. Cao and T. R. Shrout, Intrinsic and extrinsic size effects in fine-grained morphotropicphase-boundary lead zirconate titanate ceramics, J. Am. Ceram. Soc. 81, 677 (1998). 